\title{
Variability of the Radio Nucleus of the Galaxy M81
}

\author{
M. F. Bietenholz \& N. Bartel \\ Department of Physics and Astronomy, York University, Ontario, Canada
}

M. P. Rupen

National Radio Astronomy Observatory, Socorro, NM 87801, U.S.A.

Abstract. We report on VLA and VLBI observations of the nucleus of the nearby spiral galaxy M81. The VLA observations show the flux density of the nucleus to be variable by $50 \%$. The VLBI observations indicate that the structure of the nucleus of M 81 is somewhat variable on timescales of weeks.

\section{Introduction}

$\mathrm{M} 81$, at a distance of $4 \mathrm{Mpc}$, is the nearest galaxy with an active galactic nucleus, with the possibly exception of Cen $\mathrm{A}$. The nucleus is exceptionally compact: $700 \mathrm{AU} \times 300 \mathrm{AU}$ at $22 \mathrm{GHz}$, with the size being proportional to $\nu^{-0.8}$ (Bietenholz et al. 1996; Bartel et al. 1995; Bartel et al. 1982; see also Kellermann et al. 1976). Here we present further results from new VLA and VLBI observations of the nucleus of M81.

\section{VLA Flux Density Monitoring}

The nucleus of $\mathrm{M} 81$ was used as a reference source for the continuing program of VLBI monitoring of SN 1993J (Rupen et al., these Proceedings, p. 355). VLA data were taken simultaneously with the VLBI runs. Flux densities were derived from images, and the results are shown in Figure 1. The systematic uncertainty in the flux calibration (5\%) dominates internal uncertainties. The flux density of M81 varies by over a factor of 2 at $8.4 \mathrm{GHz}$ (Van Dyk \& Ho, these Proceedings, p. 205). At this frequency, the mean observed flux density was $120 \mathrm{mJy}$ with a standard deviation of $22 \mathrm{mJy}$ or $19 \%$. The flux densities at 5 and $8.4 \mathrm{GHz}$ seem well correlated, although the radio spectral index $\left(\alpha\right.$, where $\left.S \propto \nu^{\alpha}\right)$ is not constant within our errors: $\alpha$ varies by up to $3.2 \sigma$.

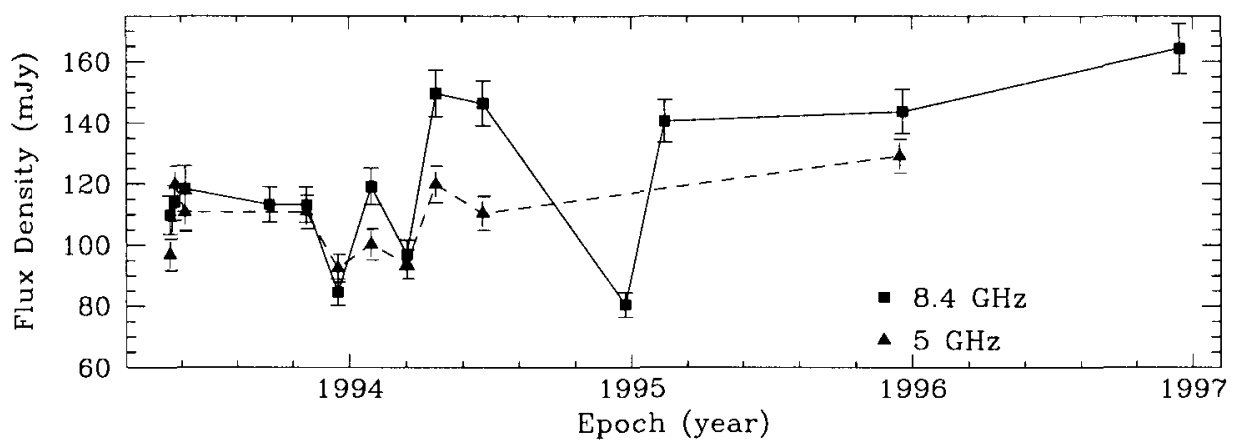

Figure 1. VLA flux density measurements 
Bietenholz, Bartel, \& Rupen

Table 1. Summary of the VLBI Model-Fitting Results at $8.4 \mathrm{GHz}$

\begin{tabular}{|c|c|c|c|c|c|c|c|c|c|c|}
\hline \multirow{4}{*}{ Date } & \multirow{4}{*}{$S$} & \multirow{2}{*}{\multicolumn{3}{|c|}{$\begin{array}{l}\text { One Component Fit } \\
\text { Elliptical Gaussian }\end{array}$}} & \multicolumn{6}{|c|}{ Two Component Fit } \\
\hline & & & & & \multirow{2}{*}{\multicolumn{2}{|c|}{$\frac{\text { Elliptical }}{\text { Axes }}$}} & \multirow{3}{*}{ P.A. } & \multicolumn{3}{|c|}{ Point Source } \\
\hline & & \multicolumn{2}{|c|}{ Axes } & \multirow[t]{2}{*}{ P.A. } & & & & \multirow[t]{2}{*}{$\%$ of $S$} & \multirow[t]{2}{*}{$r$} & \multirow[t]{2}{*}{$\theta$} \\
\hline & & Maj. & Min. & & Maj. & Min. & & & & \\
\hline $\mathrm{dd} / \mathrm{mm} / \mathrm{yy}$ & $\mathrm{m} J \mathrm{y}$ & mas & mas & ${ }^{\circ}$ & mas & mas & 0 & & mas & ${ }^{\circ}$ \\
\hline $16 / 05 / 93$ & $\overline{114}$ & 0.44 & 0.17 & 58 & 0.40 & 0.18 & 57 & 5 & 0.57 & 74 \\
\hline $16 / 12 / 93$ & 85 & 0.44 & 0.21 & 50 & 0.42 & 0.17 & 46 & 5 & 0.61 & 81 \\
\hline $21 / 06 / 94$ & 146 & 0.61 & 0.18 & 45 & 0.48 & 0.17 & 41 & 14 & 0.58 & 51 \\
\hline $12 / 02 / 95$ & 141 & 0.44 & 0.19 & 50 & 0.44 & 0.13 & 50 & 6 & 0.87 & 45 \\
\hline \multirow[t]{2}{*}{$18 / 12 / 95$} & 144 & 0.43 & 0.23 & 49 & 0.40 & 0.17 & 61 & 13 & 0.62 & 57 \\
\hline & & \multicolumn{3}{|c|}{ approximate standard errors } & 0.05 & 0.05 & 5 & 2 & 0.10 & 10 \\
\hline
\end{tabular}

Notes: $S$ are flux densities from VLA observations at $8.4 \mathrm{GHz}$; axis sizes are FWHM.

\section{VLBI Imaging and Modelfitting}

In our VLBI observations, the nucleus of M81 is only slightly resolved and somewhat asymmetrical (in agreement with Bietenholz et al. 1996). In Table 1, we give the results of fitting simple geometrical models to fully calibrated $u-v$ data.

We used the AIPS program OMFIT to simultaneously fit model parameters and determine the complex antenna gains. In the simplest case of fitting a single elliptical Gaussian, the size varies by $40 \%$. The data, however, are not well described by such a model: in all cases, the fit is significantly improved if we fit, in addition to the central elliptical Gaussian source, a weaker point source at relative position $(r, \theta)$. Though our resolution is inadequate to determine the detailed source structure, the data clearly demand structure more complicated than a single elliptical Gaussian. Note that our conservative estimates of the uncertainties take into account any contribution from the fit time-variable antenna gains (a more complete description will be published elsewhere).

In summary, we find that there is structure on scales of $\sim 0.5$ mas in the nucleus of M81. It can be described as an elongated core, with a north-east south-west orientation, and a component of lower flux density to the northeast thereof. This latter component, in particular, appears to be variable on timescales of weeks.

Acknowledgments. Research at York University was partly supported by NSERC. The National Radio Astronomy Observatory is a facility of the National Science Foundation, operated under a cooperative agreement by Associated Universities, Inc.

\section{References}

Bartel, N., et al. 1982. ApJ, 262, 556-563.

Bartel, N., Bietenholz, M. F., \& Rupen, M. P. 1995. Proc. Natl. Acad. Sci. USA, 92, 1137411376.

Bietenholz, M. F., et al. 1996. ApJ, 457, 604-609.

Kellermann, K. I., et al. 1976. ApJ, 210, L121-122. 